\title{
Preliminary Study on Improving Resolution of D-T Neutron Radiography based on Associated Alpha and Coded Source Imaging Methods
}

\author{
Sheng Wang*, Yang Wu, Heyong Huo, Hang Li, Chunlei Wu, Li An, Bin Tang, Zhenghong Li \\ * Institute of Nuclear Physics and Chemistry, CAEP \\ mritn5851@gmail.com
}

\begin{abstract}
Limitations of fast neutron radiography include low detection efficiency and poor spatial resolution. D-T neutron radiography is one compact fast neutron radiography method. Based on D-T associated alpha particle method and coded source imaging method, we indicate one new method to improve resolution of D-T neutron radiography. This method could get distribution of D-T neutrons by detecting alpha particles. Without real coded mask, the D-T radiography structure is considered as coded source imaging of fast neutrons. With reconstruction method, the real object could be reconstructed from projections. One prospect setup of D-T associated alpha neutron source has been carried out with Monte-Carlo simulation. The projection images of two different situations are collected and reconstruction results show that it's possible to improve image quality of D-T neutron radiography.
\end{abstract}

Keywords-D-T Neutron Radiography, Associated Alpha Method, Coded Source Imaging Method, Reconstruction

\section{INTRODUCTION}

$\mathrm{N}$ EUTRON radiography (NR) is one of the most important nondestructive testing methods, which is sensitive to low density materials. Especially, D-T Neutron radiography could penetrate heavy shielding materials of large thickness and distinguish flaws of low density materials $[1,2]$. Traditional D-T neutron radiography could supply L/D ratio with several hundred limited by the size of neutron source and distance between source and detector, which restricts the resolution of D-T neutron radiography.

Based on the associated alpha particles of D-T source, one could acquire distribution of D-T neutron source with a small pinhole, and then subdivide the whole neutron source into smaller neutron sources, which could improve the L/D ratio with several multiples. With method of coded source imaging (CSI) [3-6], then one could reconstruct images from raw projections.

This method is similar as associated particle imaging (API) [7, 8], but it's not interrogation method based on time of flight of D-T neutrons and scattering neutrons. In this method, one needs an alpha particle detector to collect alpha particle

Manuscript received July 4, 2019. This work was supported by National Natural Science Foundation of China (Grant no. 11605168).

Sheng Wang is with Institute of Nuclear Physics and Chemistry, CAEP, Mianyang of Sichuan Prov., 621900, China. (telephone: 00860816249 4238, e-mail: mritn5851@gmail.com). distribution without time detection ability. The neutron detector only need position detection ability too. It means that requirement of detectors could be simpler than API method.

We indicate principle of this method, and carry out Monte-Carlo simulation with a glancing incidence deuterium beams, getting the distribution of D-T neutron sources and reconstruction results from projection images. The results show that it's possible to improve image quality of D-T neutron radiography.

\section{PRINCIPLE}

The idea of this method is shown in Fig. 1. With a pinhole between D-T neutron source and Alpha particle detector, one could collect the distribution of alpha particles, which means that the distribution of D-T neutron could be recorded. Then neutron source is subdivided as multi smaller neutron sources. The projection of D-T neutron radiography could be collected by one neutron imaging detector. The projection is convolution of neutrons distribution and object distribution. With reconstruction method, we could get the object's reconstruction images from projection images.

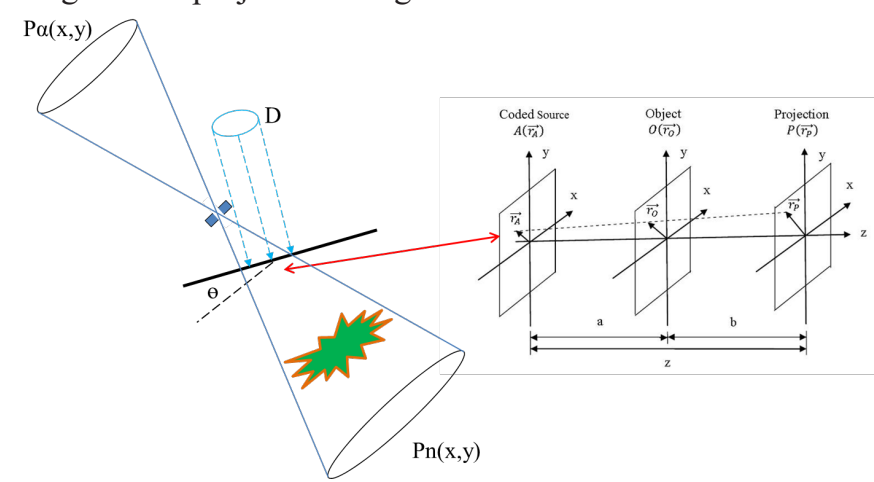

Fig. 1. The principle of this method

\section{D-T NEUTRON DISTRIBUTION}

Based on theory analysis we could get the relation as follow:

$$
\begin{gathered}
\frac{d_{\alpha}}{d_{h}}=\frac{x}{y} \\
l_{\alpha}=x+y \\
\frac{d_{h}}{d_{s}}=\frac{y}{y+l_{s}}
\end{gathered}
$$


Where $\mathrm{D}$ beam incidence angle is $\theta_{s}$, pinhole diameter is $d_{h}$, alpha detector resolution is $d_{\alpha}$. The distance between alpha detector and pinhole as is $l_{\alpha}$, and distance between pinhole and neutron source is $l_{s}$.

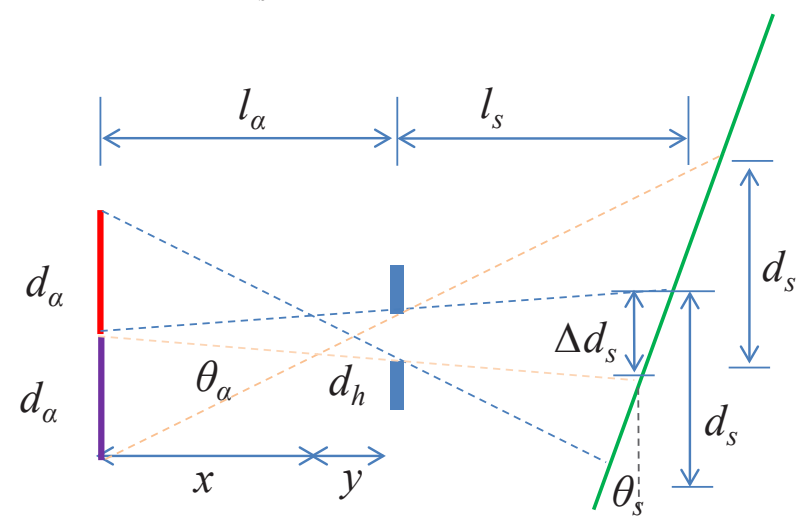

Fig. 2. The sketch map of neutron source resolution calculation

The resolution $d_{s}$ of neutron source as Eq. (4), and difference $\Delta d_{s}$ as Eq. (5).

$$
\begin{gathered}
d_{s}=d_{h}+\frac{l_{s}}{l_{\alpha}}\left(d_{\alpha}+d_{h}\right) \\
\Delta d_{s}=d_{h} \frac{l_{\alpha}+l_{s}}{l_{\alpha}}
\end{gathered}
$$

With Monte-Carlo simulation, we have calculated D-T neutron source distribution, with $\mathrm{D}$ beam as diameter $10 \mathrm{~mm}$ and incidence angle $\theta_{s}$ as 20 degrees, pinhole diameter $d_{h}$ as $0.1 \mathrm{~mm}$, alpha detector resolution $d_{\alpha}$ as $1 \mathrm{~mm}$. The distance $l_{\alpha}$ between alpha detector and pinhole is $10 \mathrm{~cm}$, and distance $l_{s}$ between pinhole and neutron source is $5 \mathrm{~cm}$. Under conditions above, the largest distance differ between $\mathrm{T}$ target and $\alpha$ detector is $10 \mathrm{~mm} \times \sin 20^{\circ} \approx 3.4 \mathrm{~mm}$, compared with total 150 $\mathrm{mm}$ could been ignored. The slope of $\mathrm{T}$ target will affect much smaller between $\mathrm{T}$ target and neutron detector.

The resolution $d_{s}$ of neutron source is $0.65 \mathrm{~mm}$ as Eq. (4), and difference $\Delta d_{s}$ is $0.15 \mathrm{~mm}$ as Eq. (5). The image of neutron source is shown in Fig. 3. It has improved the L/D ratio almost 15 times. Especially, in this setup, one single pixel of neutron is $0.65 \mathrm{~mm} \times 0.65 \mathrm{~mm}=0.4225 \mathrm{~mm}^{2}$, which include parts belonging to itself with $0.35 \mathrm{~mm} \times 0.35 \mathrm{~mm}=0.1225 \mathrm{~mm}^{2}$ and parts sharing with adjacent pixels with $0.15 \mathrm{~mm} \times 0.5$ $\mathrm{mm} \times 4=0.3 \mathrm{~mm}^{2}$. In this paper we assume neutron distribution is even, and the affection is small. But for other neutron distribution, one needs to decrease diameter of mask to get a smaller $\Delta d_{s}$.

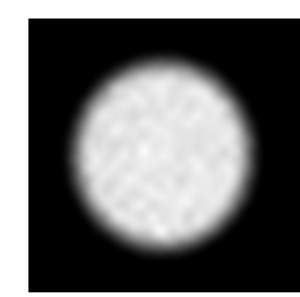

a)

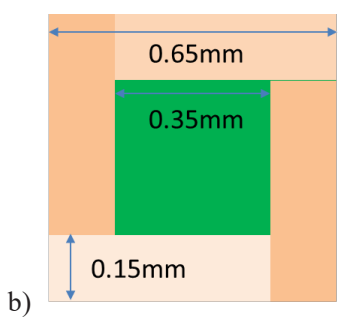

b)

Fig. 3. The distribution of D-T neutron source, a) Neutron distribution, b) Pixel distribution

\section{CODED SOURCE IMAGING}

The traditional NR images will choose a suitable distance between neutron source and neutron detector when the size of neutron source has been determined, which could supply enough neutron flux at imaging plane. CSI could increase L/D ratio by subdivide one big neutron source into smaller neutron sources [9]. It is shown in Fig. 4. The object is compressed into 2D distribution as an idea matrix.

The projection of CSI is a matrix with neutron source distribution and object distribution as follow:

$$
P=A \otimes O
$$

Where $P$ is the matrix of projection, $A$ is the neutron distribution, $O$ is the object matrix, $\otimes$ is the symbol of convolution.

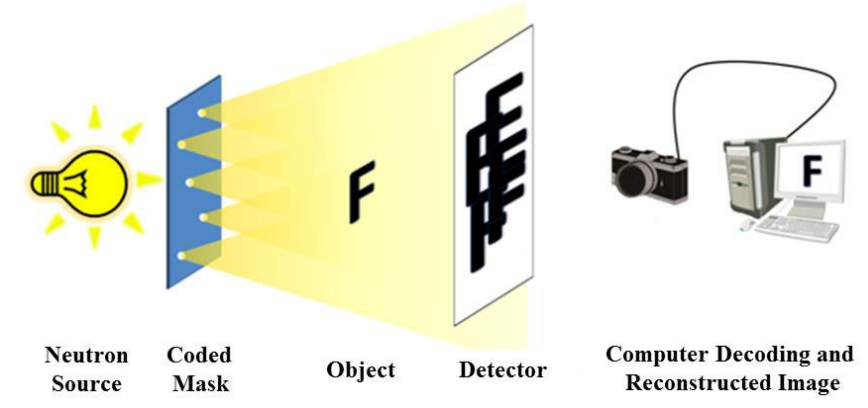

Fig. 4. The sketch map of CSI

If the object is too large, the fro and back of object couldn't been considered in on pixel at neutron. According the analysis result [10], the pixel zoom as $\omega, a, b$ and $z$ as Fig. 1, the largest object thickness differs from center is $d a$, as follow:

$$
\frac{d \omega}{\omega}= \pm \frac{z}{a b} d a
$$

Three usually used reconstruction methods of correlation reconstruction method, Wiener filter deconvolution method and maximum likelihood iteration method $[5,6]$, could be used for coded source imaging. Considering that neutron distribution matrix is much smaller than object matrix in this work, and correlation reconstruction need the mask satisfy correlation requirement [11], we only use Wiener filter deconvolution method and maximum likelihood iteration method.

Wiener filter deconvolution method is shown in Eq. (8). $C_{\text {wiener }}$ is a constant value relating with the noise level, FT and $F T^{-1}$ is Fourier transform and inverse Fourier transform, and the equation is point-wise [5].

$$
O_{\text {deco }}=F T^{-1}\left\{\frac{F T(P)}{F T(A)} \cdot \frac{|F T(A)|^{2}}{|F T(A)|^{2}+C_{\text {wiener }}}\right\}
$$

Maximum likelihood iteration method of Richardson-Lucy is shown in Eq. (9) [5].

$$
O_{\text {guess }}^{\text {new }}(x, y)=O_{\text {guess }}^{\text {old }}(x, y) \cdot \frac{P(x, y) \otimes A(-x,-y)}{\left[A(x, y) \otimes O_{\text {guess }}^{\text {old }}(x, y)\right] \otimes A(-x,-y)}
$$

\section{Monte-Carlo Simulation}

The projection of D-T neutron radiography also has been 
calculated with neutron field as $5.0 \times 10^{10} \mathrm{~s}^{-1}$, acquiring time as 10 seconds when detector efficiency is $10 \%$, neutron detector resolution as $1 \mathrm{~mm}$, distance between neutron source and detector as $1 \mathrm{~m}$.

The ideal object is composed of polyethylene and iron as Fig. 5, with different size of dots (diameter of $1 \mathrm{~mm}, 2 \mathrm{~mm}, 4 \mathrm{~mm}, 6$ $\mathrm{mm}, 8 \mathrm{~mm}$ and $10 \mathrm{~mm}$ ) and slits (width of $1 \mathrm{~mm}, 2 \mathrm{~mm}, 3 \mathrm{~mm}$, $4 \mathrm{~mm}, 5 \mathrm{~mm}$ and $10 \mathrm{~mm}$ ) in the polyethylene part, thickness as $1 \mathrm{~cm}$ and length as $8 \mathrm{~cm}$. The thickness of iron part is $3 \mathrm{~cm}$, and the length is $10 \mathrm{~cm}$. The total object is enwrapped by one square diaphragm with thickness as $10 \mathrm{~cm}$, inside length as $10 \mathrm{~cm}$ and outside length as $20 \mathrm{~cm}$.

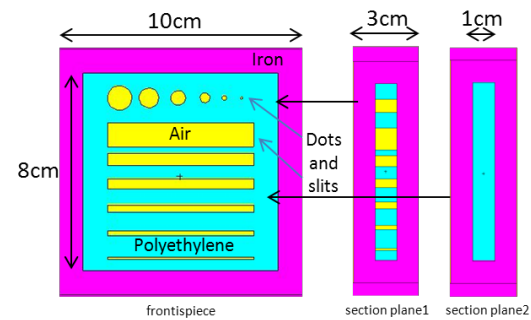

Ideal Object

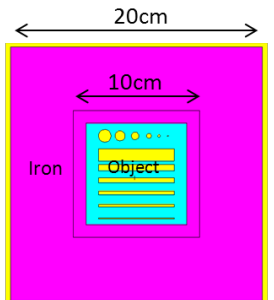

Object and Square Diaphragm
Fig. 5. The sketch map of object

Based on the resolution of neutron source and detector, distance between object and detector is determined as [6]. The distance $b$ between object center and detector should be $60.6 \mathrm{~cm}$. The projection image could be calculated with Monte-Carlo simulation, it size is $130 \times 130$. With Eq. (7), supposed that $d \omega / \omega$ is $\sqrt{0.001} \approx 3.16 \%$, with total distance as $1000 \mathrm{~mm}$, pixel of neutron as $0.65 \mathrm{~mm}$, pixel of detector as 1 $\mathrm{mm}$, then $d a$ is about $7.5 \mathrm{~mm}$. The total thickness of object could be $15 \mathrm{~mm}$, which is larger than light materials of object.

Based on deconvolution and maximum likelihood iteration method of Richardson-Lucy, we could get the reconstruction image from projection image. The reconstruct images show that distribution of dots could be distinguished by this method, and image quality could be improved from projection image as Fig. 6.

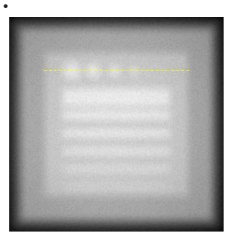

Projection

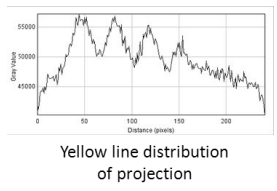

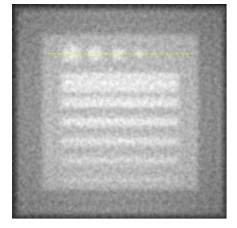
Deconvolution

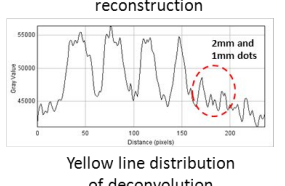

of deconvolution

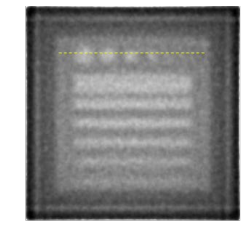
Iteration reconstruction

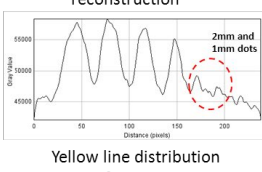

of line distribution
of iteration
Fig. 6. The results of Monte-Carlo simulation when distance between object and detector as $60.6 \mathrm{~cm}$

Compared with traditional D-T neutron radiography, the distance between object and neutron detector is much farther. It will extend projection images and impair L/D ratio improvement. We could subdivide the projection pixel into smaller pixel to fulfill requirement of coded source imaging.
On condition that distance between object center and detector as $3 \mathrm{~cm}$, pixel of neutron detector should be $0.02 \mathrm{~mm}$. With calculation resolution of neutron detector as $1 \mathrm{~mm}$, projection image matrix will be enlarged for 50 times. The other conditions are same as above. Two setups is shown in Fig. 7.

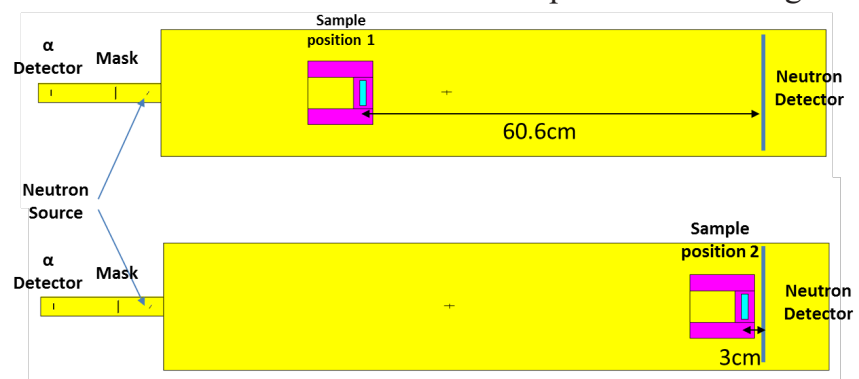

Fig. 7 Two setups of geometer

The projection and reconstruct images are shown in Fig. 8 . With newly method of reconstruction, the image's quality also improves. Limited by calculation speed, SNR of projection images is not so high enough, and reconstruction images could be better when we increase account of neutrons.

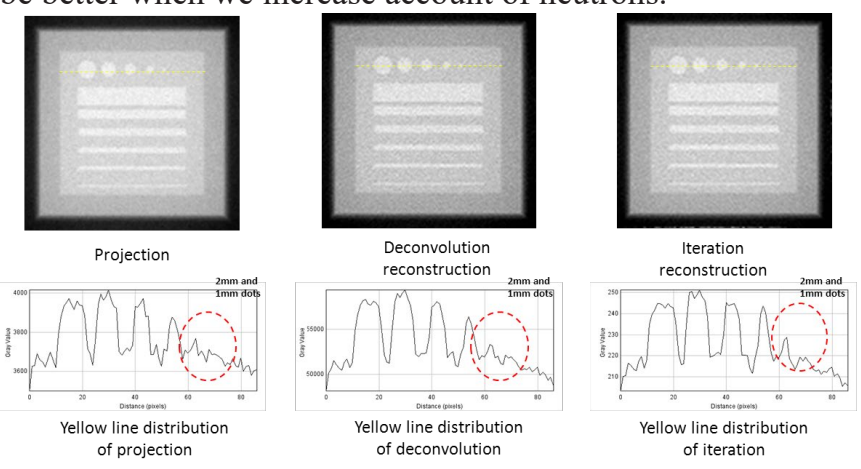

Fig 8. The results of Monte-Carlo simulation when distance between object and detector as $3 \mathrm{~cm}$

\section{CONCLUSION}

With theory analysis and Monte-Carlo simulation, we indicate one new method to improve D-T neutron radiography quality. With associated alpha particle distribution detection and D-T neutron projection images detection, we could get the reconstruction results of object distribution by coded source imaging method. The key component of this method is an associated alpha particle D-T neutron source. The neutron detection could use common neutron imaging detectors. It supply one possibility to improve L/D ratio of D-T neutron radiography with more than 10 times. On the other side, keeping $\mathrm{L} / \mathrm{D}$ ratio as traditional D-T neutron radiography, we could enlarge size of D-T neutron source by this method. It will decrease power intensity in $\mathrm{T}$ target, which is beneficial to improving neutron source lifetime and neutron flux.

This paper only analysis the ideal principle and carry out Monte-Carlo simulation, it need much more work to push it into application, such as $\alpha$ detector and neutron target structure improvement. On the other hand, this method still has some limitations. The CSI structure limited the testing size of object, and $\alpha$ detection for neutron distribution increase the noise, it need more analysis and simulation to estimate its feasibility. 


\section{ACKNOWLEDGE}

This work was financially supported by National Natural Science Foundation of China (Grant no. 11605168).

\section{REFERENCES}

[1] B. Tang, "The plot experimental study of $14 \mathrm{MeV}$ fast neutron digital radiography," Chinese Science, vol. 52, no. 9, pp. 1330-1336, 2009.

[2] B. Tang, "The physics analysis and experiment study of zinc sulphide scintillator for fast neutron radiography," Nucl. Ins. and Meth. A, vol. 729, no. 327-333, 2013.

[3] A. L. Damato, "Coded Source Imaging for Neutrons and X-Rays,” IEEE NSS, vol. 11, no. 6, pp. 199-203, 2006.

[4] A. L. Damato, "Progress Report on a Neutron Coded Source Phase Contrast Imaging System at the MIT Reactor," IEEE NSS, vol. 22, no. 440, pp. 1725-1728, 2007.

[5] F. Grünauer, "Design, Optimization, and Implementation of the New Neutron Radiography Facility at FRM-II," Techische Universitat Munchen, Munich, Germany, 2005.

[6] S. Wang, "Coded source imaging simulation with visible light”, Nucl. Ins. and Meth. A, vol. 65, pp. 187-191, 2011.

[7] J.T. Mihalczo, "Physical description of nuclear materials identication system (NMIS) signatures," Nucl. Ins. and Meth. $A$, vol. 450 , pp. 531-555, 2000 .

[8] A. J. Antolak, "Negative ion-driven associated particle neutron generator," Nucl. Ins. and Meth. A, vol. 806, pp. 30-35, 2016.

[9] S. Wang, "Preliminary Study of Nuclear Fuel Element Testing Based on Coded Source Neutron Imaging," ANIMMA 2015 Conference.

[10] S. WANG, "Study on Coded Source Neutron Imaging Technology based on a Compact Accelerator," Peking University, Beijing, China, 2013.

[11] A. L Damato, "Capabilities and limitation of phase contrast imaging techniques with X-rays and neutrons," Massachusetts Institute of Technology, Cambridge, USA, 2001. 